\title{
Parameter Optimization of Linear Phased Array Transducer for Defect Detection
}

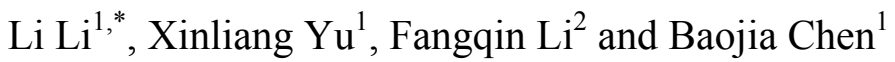 \\ ${ }^{1}$ Hubei Key Laboratory of Hydroelectric Machinery Design \& Maintenance, China Three Gorges University, Yichang, \\ 443002, China \\ ${ }^{2}$ Department of Electrical and Computer Engineering (ECE), National University of Singapore, 21 Lower Kent Ridge \\ Road, 119077, Singapore
}

\begin{abstract}
An optimization design method was proposed for linear phased array ultrasonic imaging testing. In the method, main lobe width and the amplitude ratio of side lobe to main lobe were served as optimization objectives to judge the testing precision of phased array beam directivity. The combination of element of width and inter-element space was served as optimize parameter for the optimization objectives. The combination of optimal parameters for phased array transducer was finally achieved and applied to phased array B-mode imaging ultrasonic testing on a flat bottom hole in standard test block. The results show that the method can not only obtained the optimal parameters for linear array of phased array transducer, but also improve the accuracy of the location and size for defect detection.
\end{abstract}

Keywords: Defect detection, linear phased array transducer, parameter optimization, phased array ultrasonic B-mode imaging testing.

\section{INTRODUCTION}

Due to the flexibility of control of ultrasonic beam directivity and the size and location of focus, phased array ultrasonic imaging testing technology plays a critical role in defect detection among aerospace, railway, shipping and nuclear industry [1-5]. The resolution of phased array ultrasonic imaging is one of the most concerned of these industrial detection fields. According to the research, phased array transducer parameters are essential to obtain a high ultrasonic imaging resolution [6-8]. Improper parameters of phased array transducer would lead to a poor phased array ultrasonic beam directivity, and then cause defect resolution artifacts, grating lobe or side lobe effect artifacts in the ultrasonic imaging $[9,10]$. Artifact means that the position of echo signal in ultrasonic imaging testing system does not agree with the actual position in the echo interface for the testing body, or the displayed signal amplitude and the change of gray scale does not relate to the variations of echo interfacial characterization $[9,10]$. Any artifact can greatly deteriorate the accuracy of the defect detection. Therefore, it is crucial to establish an appropriate set of design parameters for phased array transducer for obtaining accurate defect ultrasonic imaging and defect diagnosis [8].

It is noticed that the current method for a linear phased array transducer parameters selection (called "current method") is only focused on analyzing or optimizing for single parameter. It does not provide optimal matching parameters for a linear phased array transducer [11]. Therefore an optimization design of a linear phased array parameters method was presented in this paper. The normalized main lobe width and the amplitude ratio of the side lobe to main lobe were served as optimization objectives to judge the testing precision of phased array beam directivity. Element width $a$ and inter-element spacing $d$ were served as optimized parameters for the optimization objectives. The combination of optimal parameters for phased array transducer was finally achieved by analyzing and applied to phased array B-mode imaging ultrasonic testing on a flat bottom hole in standard test block. The results show that the method can not only obtained the optimal parameters for linear array of phased array transducer, but also improve the accuracy of the location and size for defect detection.

\section{BEAM DIRECTIVITY FOR LINEAR PHASED ARRAY TRANSDUCER}

Beam directivity for linear phased array transducer descrips acoustic pressure amplitude with the variation of azimuth angle [6-8]. Linear phased array transducer consists of $\mathrm{N}$ individual elements. Its beam directivity can be defined as following.

$$
D(\theta)=\left|\frac{\sin \left[\frac{k N d}{2}\left(\sin \theta-\sin \theta_{s}\right)\right]}{N \sin \left[\frac{k d}{2}\left(\sin \theta-\sin \theta_{s}\right)\right]}\right| \cdot\left|\frac{\sin \left(\frac{k a}{2} \sin \theta\right)}{\frac{k a}{2} \sin \theta}\right|
$$

Where, 


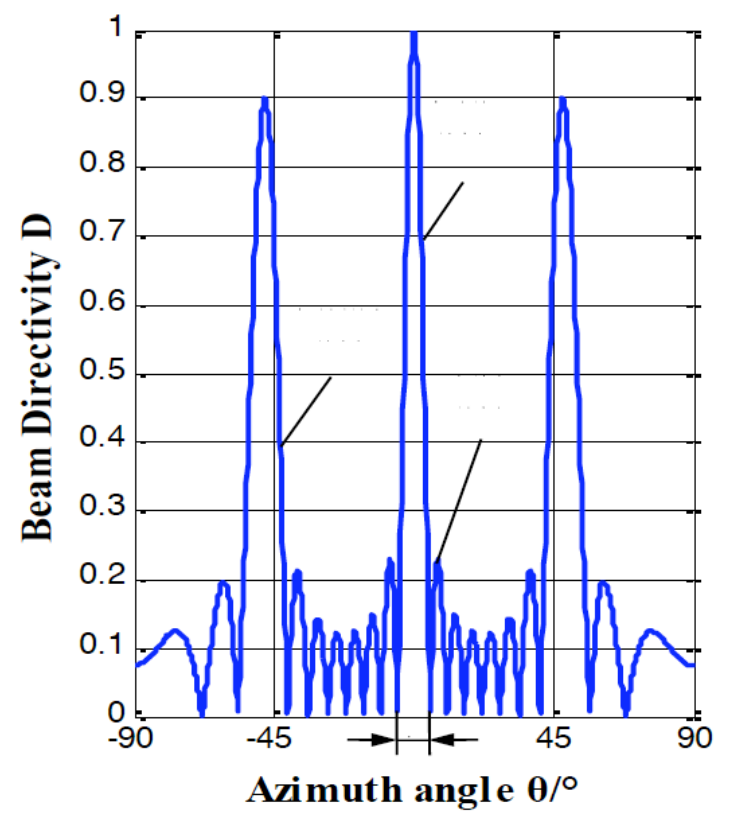

Fig. (1). Beam directivity of linear phased array transducer.

$a$--- element width;

$d$--- inter-element space;

$k=2 \pi / \lambda$;

$\lambda$--- wave length;

$f$--- operating frequency for transducer;

$\theta$--- azimuth angle;

$\theta_{s}$--- steering angle.

Beam directivity consists of main lobe, grating lobe and side lobe, shown in Fig. (1). Well beam directivity can be achieved by minimizing the main lobe width, eliminating grating lobes and suppressing side lobes, so that its ultrasonic beam can be steered and focused on whole testing area. The parameters $(N, a)$ have a great effect on the beam directivity.

\subsection{Influence of Transducer Parameters on Beam Directivity}

\subsubsection{Influence on Main Lobe}

As shown in in Fig. (1), main lobe width $q$ is defined as the distance of main lobe on the azimuth angle $\theta$ axis

$q=\frac{1}{\pi}\left[\sin ^{-1}\left(\sin \theta_{s}+\frac{\lambda}{N d}\right)-\sin ^{-1}\left(\sin \theta_{s}-\frac{\lambda}{N d}\right)\right]$

Relationships between $q, N$ and $\lambda$ are shown in Fig. (2). In Fig. (2a) main lobe width q decreases with both element number $N$ and inter-element space $d$. Since smaller $q$ will lead to a higher ultrasonic imaging resolution, phased array beam directivity can be improved by increasing element number $N$ and inter-element space $\mathrm{d}$.

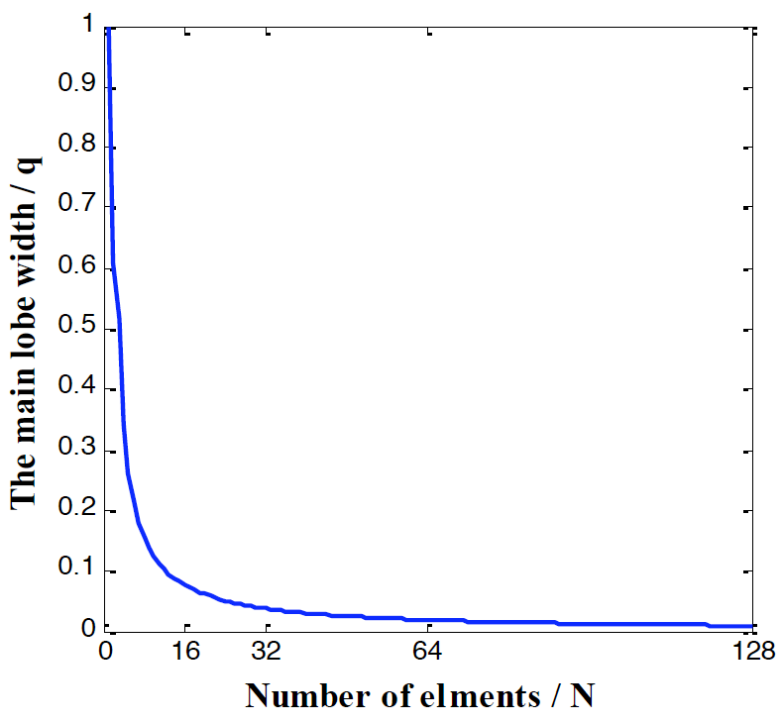

(a)

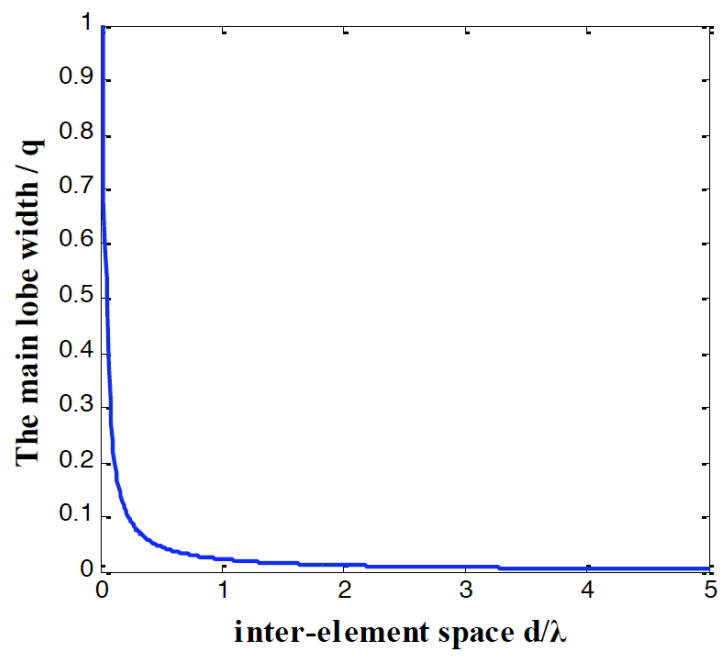

(b)

Fig. (2). Influence of linear phased array transducer parameters on the main lobe $\theta_{0}=30^{\circ}, f=10 \mathrm{MHz}, c=5940 \mathrm{~m} / \mathrm{s}$ (a) Influence of elements number $N$ on the main lobe width $q(d / \lambda=0.6)(\mathbf{b})$ Influence of inter-element space $d / \lambda$ on main lobe width $q(N=32)$.

\subsubsection{Influence on Grating Lobe}

The appearance of grating lobe will lead to both energy leakage and spurious signal. If inter-element space $d$ and wavelength $\lambda$ satisfy inequality (3), grating lobe would not appear in linear phased transducer beam directivity diagram and grating lobe effect artifact [6-8].

$\frac{d}{\lambda} \leq \frac{(N-1)}{N\left(1+\sin \theta_{s}\right)}$

\subsubsection{Influence on Slide Lobe}

Side lobes are the small lobes near to main lobe in beam directivity diagram shown in Fig. (1). The ratio of side lobe to main lobe $\varepsilon$ is as follows. 


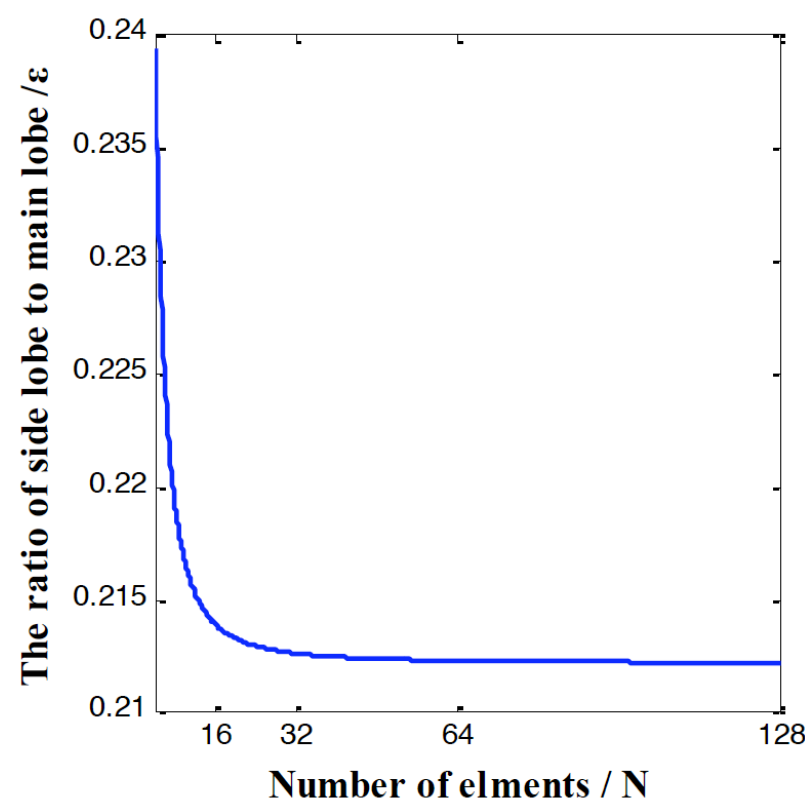

(a)

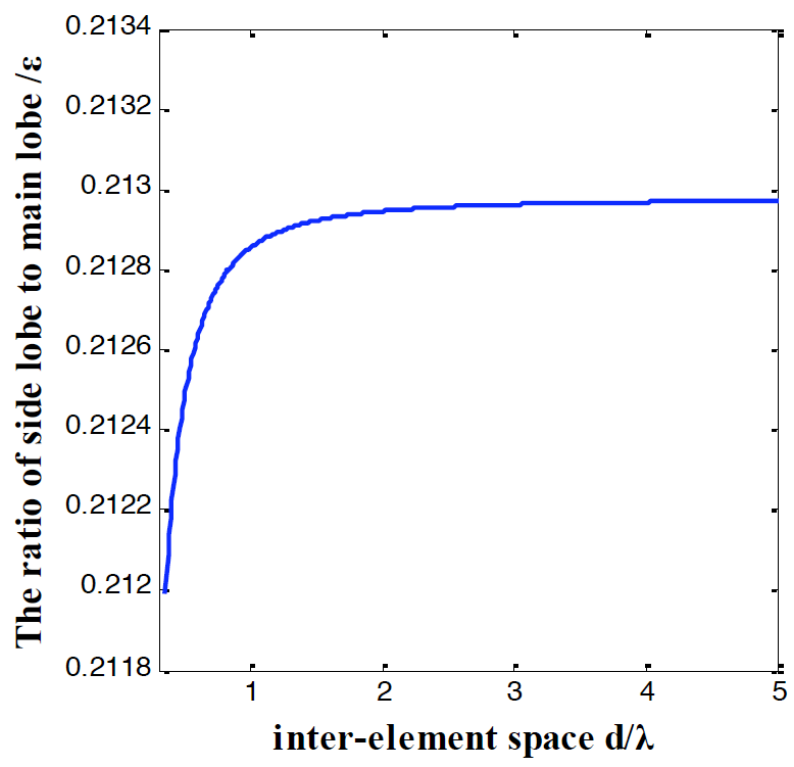

(b)

Fig. (3). Influence of linear phased array transducer parameters on side lobe $\theta=0^{\circ}, f=10 \mathrm{MHz}, c=5940 \mathrm{~m} / s, a / \lambda=0.35$ (a) Influence of element number $N$ on main lobe width $q(d / \lambda=0.6)$ (b) Influence of inter-element space $d / \lambda$ on main lobe width $q(N=32)$.

$\varepsilon=\left|\frac{\sin \left[\frac{k N d}{2}\left(\sin \theta-\sin \theta_{s}\right)\right]}{N \sin \left[\frac{k d}{2}\left(\sin \theta-\sin \theta_{s}\right)\right]}\right| \cdot\left|\frac{\sin \left(\frac{k a}{2} \sin \theta\right) \cdot \frac{k a}{2} \sin \theta_{s}}{\frac{k a}{2} \sin \theta \cdot \sin \left(\frac{k a}{2} \sin \theta_{s}\right)}\right|$

where, $\theta=\frac{1}{2} \sin \theta^{-1}\left(\sin \theta_{s}-\frac{\lambda}{N d}\right)+\frac{1}{2} \sin \theta^{-1}\left(\sin \theta_{s}-\frac{2 \lambda}{N d}\right)$

It can be found in Fig. (3) that $\varepsilon$ decreases with element number $N$, while increases with inter-element space $d(d>a)$.
Therefore, bigger elements number $N$ and smaller interelement space $\mathrm{d}$ contribute to less side lobe.

\subsection{Influence of Transducer Parameters on Testing Area}

Compared to the axial direction of the steering angle of acoustic pressure, the acoustic pressure amplitude generated by the linear phased array transducer should not be lower than $6 \mathrm{~dB}$, this range is called testing area angle.

$$
\beta_{-6 \mathrm{~dB}}=2 \sin ^{-1}\left(\frac{0.6 \lambda}{a}\right)
$$

If $0.6 \lambda / a \geq 1$, testing area angle is $180^{\circ}$ and the linear phased array ultrasonic beam can be steered and focused. Therefore, the element width of a linear phased array transducer should meet the following condition.

$$
a / \lambda \leq 0.6
$$

\subsection{Analysis of Phased Array Transducer Parameters Optimization Design}

According to the analysis of beam directivity and testing area, several criterions for selection of linear phased array transducer are obtained:1) Increase element number $N$ and inter-element space $d$ to decrease the main lobe width $q ; 2$ ) Inequality (3) should be satisfied to eliminate side lobe; 3 ) Increase element number $N$ or decrease element width $a$ to suppress side lobe amplitude; 4) Inequality (6) should be satisfied to make the whole testing area covered.

\subsubsection{Variable Selection}

According to the design rules for phased array transducer parameters, element width $a$ and inter-element space $d$ play decisive roles on the beam directivity of linear phased array transducer.

$$
X=(a, d)
$$

\subsubsection{Optimization Function Design}

Artifacts produced by undesirable beam directivity affect the imaging resolution directly. In order to minimize the main lobe width $\mathrm{q}$ and suppress side lobe amplitude, the minimum main lobe width $\mathrm{q}(X)$ and the ratio of minimum side lobe to main lobe $\varepsilon(X)$ are taken as optimization functions.

$$
\left\{\begin{array}{l}
f_{1}(X)=\min q(X)=\min \left(\frac{1}{\pi}\left[\sin ^{-1}\left(\sin \theta_{s}+\frac{\lambda}{N d}\right)-\sin ^{-1}\left(\sin \theta_{s}-\frac{\lambda}{N d}\right)\right]\right) \\
f_{2}(X)=\min \varepsilon(X)=\min \left|\frac{\sin \left[\frac{k N d}{2}\left(\sin \theta-\sin \theta_{s}\right)\right]}{N \sin \left[\frac{k d}{2}\left(\sin \theta-\sin \theta_{s}\right)\right] \mid}\right| \cdot\left|\frac{\sin \left(\frac{k a}{2} \sin \theta\right) \cdot \frac{k a}{2} \sin \theta_{s}}{k a} \sin \theta \cdot \sin \left(\frac{k a}{2} \sin \theta_{s}\right)\right|
\end{array}\right.
$$

\subsubsection{Constraint Condition Design}

Grating lobe elimination and complete coverage of testing area are designed as optimal constraint conditions. 


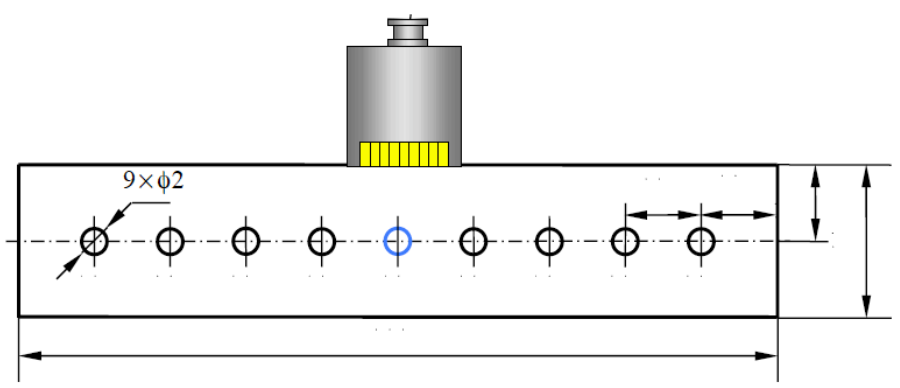

Fig. (4). Phased array imaging ultrasonic test to a flat bottom hole $5 \#$ in CSII -1/20 standard test block.

Table 1. Comparison of beam directivity for two phased array transducer.

\begin{tabular}{|c|c|c|c|}
\hline Rotation Velocity (rpm) & Current & Optimization & Comparison \\
\hline \hline Main lobe width $q$ & 0.0685 & 0.0359 & $47.59 \%$ \\
\hline Granting lobe & No & No & - \\
\hline Testing area Angle $^{\text {o }}$ & 180 & 180 & - \\
\hline
\end{tabular}

$\frac{d}{\lambda} \leq \frac{(N-1)}{N\left(1+\sin \theta_{s}\right)}, \frac{a}{\lambda} \leq 0.6$

\subsection{Application}

In order to verify the proposed method, as shown in Fig. (4), a CSII -1/20 standard test block with a flat bottom hole $5 \#$ is taken as a test object for defect. A set of reasonable parameters of a linear phased array transducer is studied. In the application, we choose $N=32, c=5940 \mathrm{~m} / \mathrm{s}, f=10$ $\mathrm{MHz}, \lambda=0.594 \mathrm{~mm}$, array aperture $D=12 \mathrm{~mm}$ and steering angle $\theta_{s}=30^{\circ}$. Employing the method, an optimized result was got as $a=0.107625 \mathrm{~mm}, d=0.383625 \mathrm{~mm}$.

\subsection{Comparative Analysis}

The results of optimal parameter design for phased array transducer $(N=32, f=10 \mathrm{MHz}, d=0.38 \mathrm{~mm}, a=0.11 \mathrm{~mm})$ are compared with the results of current method $(N=32$, $f=10 \mathrm{MHz}, d=0.20 \mathrm{~mm}, a=0.11 \mathrm{~mm})$ in Table 1 and Fig. (5).

As shown in Table 1, main lobe width and side lobe amplitude of optimal parameter designing for phased array transducer are of $47.59 \%$ and $0.42 \%$ smaller than current method respectively, but the beam directivity for both is fine.

Fig. (5) shows the phased array B-mode imaging simulation results of a flat bottom hole $5 \#$ using these two transducers [12]. After convert these two B-mode grayscale images to binary images based on threshold, accordingly, defect edges are extracted in Fig. (5c) (dash curve and full curve). We can see that the location (approximately $0.25 \mathrm{~mm}$ ) and size $(\Phi=2 \mathrm{~mm})$ of defect detection after parameter optimization (dash curve) are obviously consistent with the real position $(0.25 \mathrm{~mm}$ ) and size $\Phi=2 \mathrm{~mm}$ (bold red curve), but the current method on defect evaluation (the full curve) have a big error. It indicates that parameter optimization method can test the location and size of defect more accurately and quantitatively than current method. The information of structure defect can be distinguished by parameter optimization method. It also proves the feasibility and validity of this method.

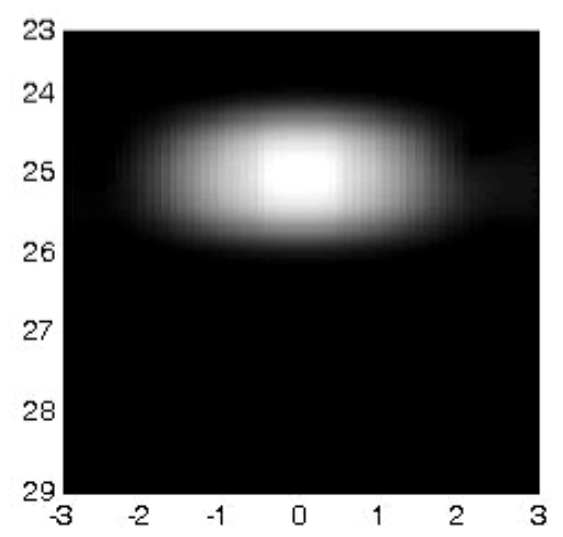

(a)

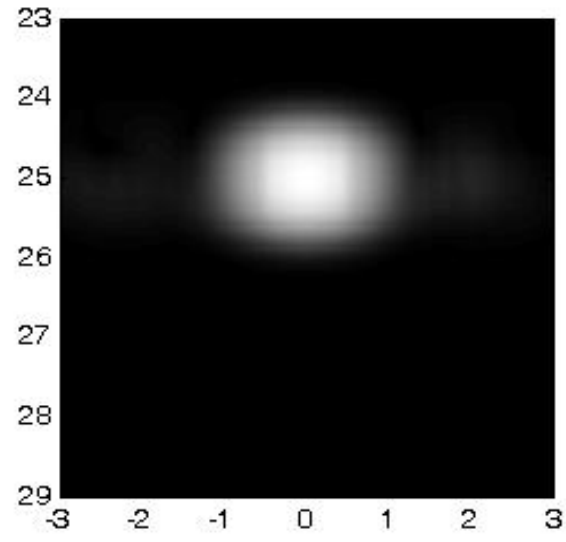

(b) 


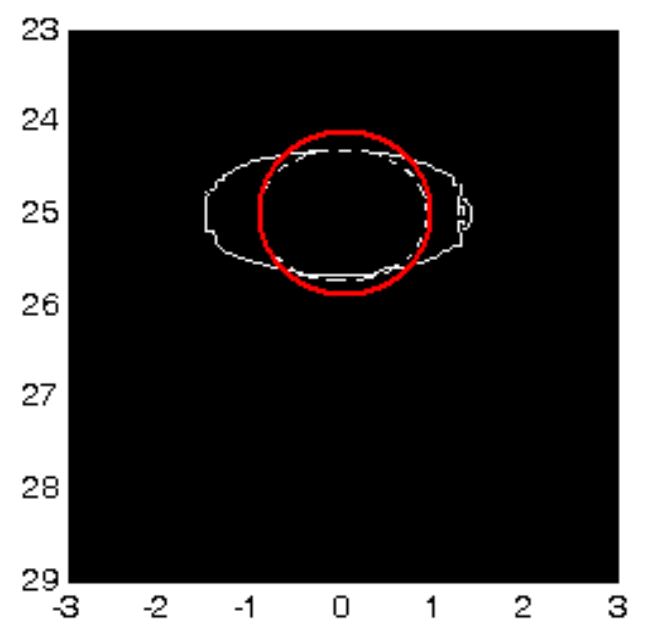

(c)

Fig. (5). Comparison of phased array B-mode imaging simulation results to a $5 \#$ flat bottom hole in CSII $-1 / 20$ standard test block (a) Current method (b) Parameter optimization (c) Comparison

\section{CONCLUSION}

Aimed at improving defect detection quality of linear phased array ultrasonic imaging test, an optimization design for a linear phased array parameters method was presented in this paper. The effectiveness of this method has been verified by a case study. It can be concluded that:

(1) A parameter optimization method for linear phased array ultrasonic has been proposed. This method is based on the analysis of beam directivity of phased array ultrasonic and selecting the minimum main lobe width and the ratio of side lobe to main lobe as optimization function, the elimination of side lobe and effective testing area as the constrain conditions, and then the main parameters of phased array, i.e. element width a and inter-element space $d$, were analysed and designed, the optimal parameters combination of phased array transducer were finally obtained.

(2) The application indicates that: the optimal parameter combination for phased array transducer obtained by the parameter optimization method is $\mathrm{N}=32, f=10 \mathrm{MHz}, \mathrm{d}=$ $0.38 \mathrm{~mm}, \mathrm{a}=0.11 \mathrm{~mm}$, the location $(0.25 \mathrm{~mm})$ and the size ( $\Phi=2 \mathrm{~mm}$ ) of the flat bottom hole $5 \#$ defect testing by the phased array B-mode imaging simulation agrees well with reality, it proves that the parameter method can really im- prove the accuracy of the location and size for defect detection.

\section{CONFLICT OF INTEREST}

The authors confirm that this article content has no conflicts of interest.

\section{ACKNOWLEDGEMENTS}

This work described in the paper was supported by National Natural Science Foundation of China (No. 51175401 and No.51205230).

\section{REFERENCES}

[1] B. W. Drinkwater, and P. D. Wilcox, "Ultrasonic arrays for nondestructive evaluation: A review," NDT and E International, vol. 39, no. 7 pp. 525-541, October 2006.

[2] H. Li, Y. Li, G. Lu, and K. Tian, "Ultrasonic phased array technology in the detection of composite materials application," Fiber Reinforced Plastics/Composites, no. 2, pp. 86-88, March 2010.

[3] X. Zhan, J. Li, Y. Zhang, S. Chen, Z. Zeng, and S. Jin "Ultrasonic phased array system applied in flaw detection of pipe girth welds," Chinese Journal of Scientific Instrument, vol. 27, no. 6, pp. 14271428, June 2006.

[4] Y. Ji, and J. Li, "Application of LX Portable Phased Array Wheel Rim Flaw Detection Device in EMU Wheelset Flaw Detection," Chinese Railways, no. 2, pp. 89-92, February 2011.

[5] S. Song, H. Shin, and Y. Jang, "Development of an ultrasonic phased array system for non-destructive tests of nuclear power plant components," Nuclear Engineering and Design, vol. 214, no. 2, pp. 151-161, 2002.

[6] Clay, S. Wooh, L. Azar, and J. Wang, "Experimental study of phased array beam steering characteristics", Journal of Nondestructive Evaluation, vol. 18, no. 2, pp. 59-71, June 1999.

[7] S. Wooh, and Y. Shi, "Optimum beam steering of linear phased arrays," Wave Motion, vol. 29, no. 3, pp. 245-265, April 1999.

[8] S. Wooh, and Y. Shi, "Three-dimensional directivity of phased steered ultrasound," Journal of the Acoustical Society of America, vol. 105, no. 6, pp. 3275-3282, 1999.

[9] K. Shi, and Y. Guo, Phased Array Ultrasonic Imaging, Higher Education Press: China, 2010.

[10] R. Feng, Principle and Design of ultrasonic diagnostic equipment. China Medical, Science and Technology Press: China, 1993.

[11] H. Jing, S. Zhou, and P. Que, "Optimization of Parameters for Linear Phased Array Transducer," Piezoelectrics and Acoustooptics, vol. 32, no. 1, pp. 51-54, February, 2010.

[12] J. A. Jensen, User's guide for the Field II program, Version 3.20. Department of Information Technology, Technical University of Denmark, 2011.

(C) Li et al.; Licensee Bentham Open.

This is an open access article licensed under the terms of the Creative Commons Attribution Non-Commercial License (http://creativecommons.org/licenses/by-nc/3.0/) which permits unrestricted, non-commercial use, distribution and reproduction in any medium, provided the work is properly cited. 